\title{
The 2017 Global Initiative for Chronic Obstructive Lung Disease Report and Practice Implications for the Respiratory Therapist
}

\author{
Michael W Hess RRT
}

\begin{abstract}
Since 1998, the Global Initiative for Obstructive Lung Disease (GOLD) has worked with health care professionals, medical researchers, and public health officials around the world to optimize the prevention, treatment, and management of COPD. GOLD has continually worked to ensure COPD management strategy recommendations are aligned with current published research. The 2017 GOLD report represents a major revision to GOLD strategy guidelines, the first such major revision in the last 5 years. As such, the 2017 report has significant implications for the diagnosis of COPD, the management of stable disease, and the treatment of exacerbations. As respiratory therapists (RTs) are front-line clinical professionals throughout the COPD continuum of care, a thorough understanding of the new GOLD recommendations for practice is critical. New recommendations regarding diagnosis, severity assessment, and both pharmacologic and nonpharmacologic treatment of COPD are presented, and suggestions for how RTs can integrate these recommendations into COPD care practices are provided. Key words: COPD; chronic bronchitis; pulmonary emphysema; bronchodilators; pulmonary rehabilitation; pharmacology; spirometry; GOLD. [Respir Care 2017;62(11):1492-1500. (c) 2017 Daedalus Enterprises]
\end{abstract}

\section{Background}

COPD continues to be a major driver of health care spending in the United States and around the world. Statistics from the World Health Organization indicate COPD is the third leading cause of mortality around the world. ${ }^{1}$ COPD is also a significant cause of disability, as the COPD diagnosis is associated with a greater likelihood of unemployment as well as eligibility for federal disability payments. ${ }^{2}$ It is estimated that, in the United States alone, COPD removes $\$ 50$ billion from the economy annually. ${ }^{3}$

\footnotetext{
Mr. Hess was a paid consultant for the Global Initiative for Chronic Obstructive Lung Disease. He contributed proofreading and copyediting support to the final report draft, but was not otherwise involved in the creation or research of the 2017 report.

Opinions in this document belong solely to Mr. Hess, and do not necessarily reflect those of the Global Initiative for Chronic Obstructive Lung Disease.

Correspondence: Michael W Hess RRT, Department of Internal Medicine, Western Michigan University Homer Stryker M.D. School of Medicine, 1000 Oakland Drive Kalamazoo, MI 49008. E-mail: michael.hess@med.wmich.edu.
}

DOI: $10.4187 /$ respcare.05402
In 1997, when it was becoming clear just how significant a burden COPD would place on global health care resources, agencies including the United States National Institutes of Health and the World Health Organization collaborated to create the Global Initiative for Chronic Obstructive Lung Disease (GOLD). Experts in pulmonology, public health, and a variety of other disciplines were assembled to improve the prevention and management of COPD through collaboration, evidence-based practice, and promotion of research. Three years later, GOLD issued its first Global Strategy for the Diagnosis, Management, and Prevention of COPD report. Over the course of the next decade, GOLD continued to update the report to reflect the continual advances in COPD research, with periodic minor revisions being issued through the GOLD website (http:// www.goldcopd.org) and major updates to the report taking place in 2007 and 2011.

By 2015, as COPD research continued to accelerate, it became clear to the GOLD Science Committee that a major revision to the GOLD recommendations was again necessary. Various GOLD committees and individual members spent the next 18 months reviewing literature and creating new sets of recommendations and strategy guidelines. The latest iteration of the GOLD Global Strategy report was formally introduced during the first-ever 
GOLD COPD Care Continuum Conference in Philadelphia, PA, on November 16, 2016, coinciding with that year's commemoration of World COPD Day. The new document contains a variety of new, evidence-based recommendations for the management of COPD in both inpatient and out-patient settings. ${ }^{4}$ The data supporting these recommendations were evaluated against GOLD's clinical evidence-assessment scheme, revised and updated for this edition.

\section{A New Paradigm for Diagnosis and Assessment}

One of the traditional hallmarks of the GOLD strategy has been to diagnose and stratify COPD cases on the basis of postbronchodilator spirometric values. A person with respiratory symptoms was officially diagnosed with COPD when the $\mathrm{FEV}_{1} / \mathrm{FVC}$ ratio was $<0.70 . .^{5}$ Once diagnosed, the patient was assigned to 1 of 4 severity categories on the basis of their $\mathrm{FEV}_{1}$ value compared to values predicted for the patient's height, gender, age, and other demographics. These categories, labeled GOLD Stages I-IV, were designed to be a simple and straightforward guide for clinicians to assess the severity of a particular patient's case and to guide the initiation of therapy. It was believed that most patients would follow an essentially progressive course where the severity of symptoms would worsen in response to the decline in $\mathrm{FEV}_{1}$ regardless of initial air flow limitation. However, since the initial GOLD report, it has been determined that while $\mathrm{FEV}_{1}$ is an important prognostic measure at the population level, it correlates poorly with health status in the individual.6,7 The 2011 GOLD revision attempted to account for this by adding basic symptom assessment using the COPD Assessment Test (CAT) and/or the modified British Medical Research Council Questionnaire (mMRC), as well as exacerbation history, to the spirometric staging. The resulting Combined Assessment used a quadrant-based system to assign patients into a severity group labeled A-D.

Unfortunately, the Combined Assessment of COPD was found to be flawed as well. Goossens and associates ${ }^{8}$ found the new model was actually less useful in predicting mortality or lung function decline than the basic spirometric staging system, although it was slightly more useful than the old paradigm for determining exacerbation risk. A similar Korean study ${ }^{9}$ also found lower accuracy using the Combined Assessment as compared to the traditional model. Han et $\mathrm{al}^{6}$ applied the Combined Assessment to the COPDGene research cohort and found that, while multifactorial assessment encouraged clinicians to view COPD cases in a more holistic and inclusive manner, the variations inherent in using multiple criteria to determine risk (in this case, $\mathrm{FEV}_{1}$-based staging and exacerbation history) introduced significant uncertainty and confusion into what was intended to be an easy-to-use tool. Furthermore, the simultaneous use of both exacerbation history and GOLD stage to determine future exacerbation risk created ambiguous classification in certain patients with significant air flow obstruction but few symptom flares.

The 2017 GOLD report addresses these concerns by completely dissociating the assessment of air flow limitation from the evaluation of symptom severity, creating a binomial taxonomy for patient classification. Formal diagnosis of COPD and assessment of air flow limitation are still accomplished through the use of spirometry. The old terminology of stages has been replaced by grades to reflect the concept that this measure is solely indicative of the severity of air flow impairment and not disease progression. Patients are placed in 1 of the 4 classic GOLD groups on the basis of this spirometry evaluation. The new assessment of symptom severity and risk adopts the same $\mathrm{ABCD}$ grouping paradigm as the original Combined Assessment, but uses only exacerbation history and either dyspnea (using the mMRC scale) or symptom evaluation (using the CAT score), with symptom burden being preferred. Users of the mMRC evaluation are cautioned to evaluate symptoms beyond simple breathlessness. In this new Individualized Assessment or Refined Combined Assessment, patients are now designated GOLD X, Category $\mathrm{Y}$ (where $\mathrm{X}$ is the air flow limitation grade and $\mathrm{Y}$ is the severity group placement) (see Fig. 1). By separating symptoms from air flow limitation, it is anticipated that clinicians will be able to more swiftly initiate appropriate treatment in cases where spirometry may not be practical. The importance of considering symptoms when diagnosing and classifying COPD is reinforced by a subtle change in the formal definition of COPD; whereas previous GOLD reports defined COPD solely in terms of persistent air flow defects, the latest definition states that COPD is characterized by persistent respiratory symptoms and air flow limitation secondary to airway and/or parenchymal disease. $^{4}$

While spirometry is still not considered appropriate for widespread screening, the new GOLD strategy does call for the use of spirometry in active case finding (the additional testing of populations already considered to be atrisk, such as those patients who have already been identified as symptomatic or as having multiple risk factors for COPD or those who have been identified through the use of a true screening tool such as CAPTURE).${ }^{10}$ In addition, the new GOLD report acknowledges that there may be a significant difference between measured air flow limitation and a patient's perception of their symptoms (such as in so-called pure emphysema, where the $\mathrm{FEV}_{1}$ may be relatively preserved because the disease processes are predominantly focused in the terminal small airways and alveoli, or the preserved ratio, impaired spirometry [PRISM] phenomenon in certain smokers). ${ }^{11}$ In these cases, the GOLD strategies call for more intensive evaluation of lung 


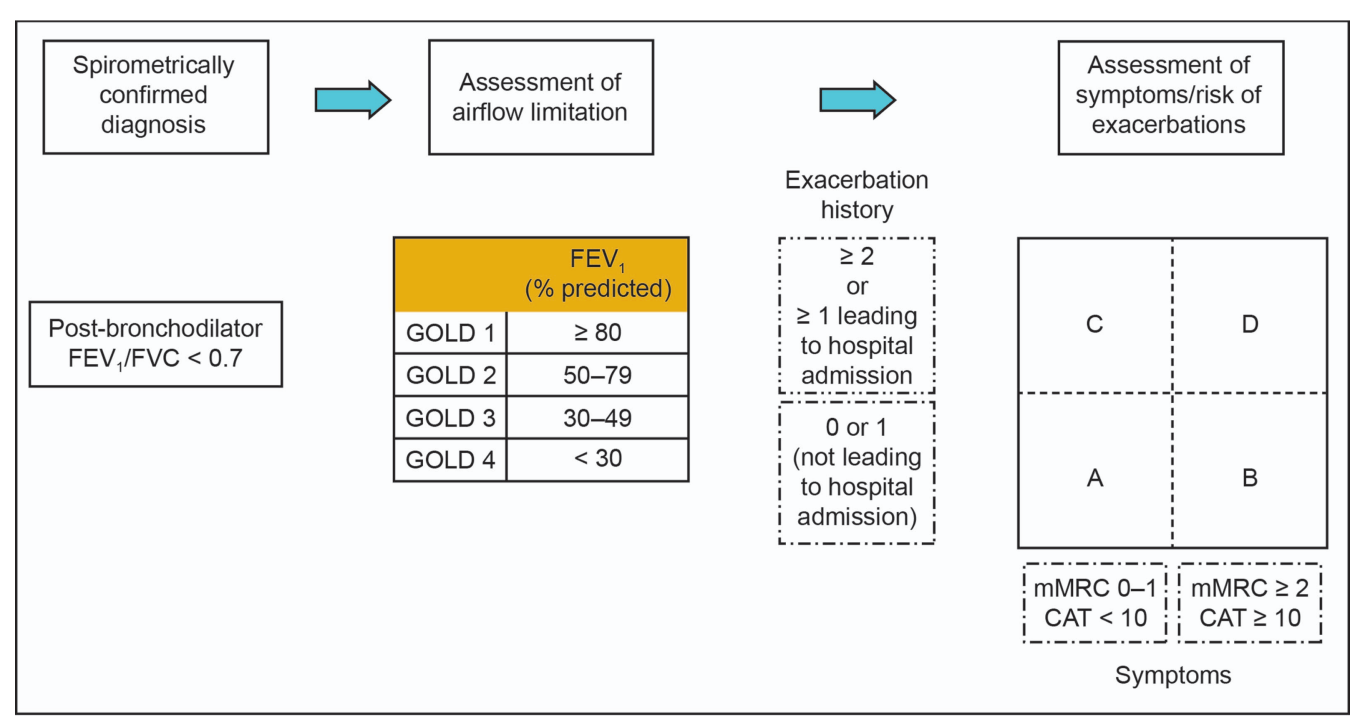

Fig. 1. Individualized assessment of COPD. GOLD = Global Initiative for Chronic Obstructive Lung Disease, mMRC = modified Medical Research Council, CAT $=$ COPD Assessment Test. From Reference 4, with permission.

mechanics, comorbidities, and other factors. GOLD's continued use of the fixed $\mathrm{FEV}_{1} / \mathrm{FVC}$ ratio over the lower limit of normal criteria may result in diagnostic error in certain populations (particularly by generating false positives for air flow obstruction in the elderly), ${ }^{12}$ reinforcing the need for detailed symptom analysis.

\section{Review of New Therapeutic Options and Recommendations}

The 2017 GOLD report takes a comprehensive look at both pharmacologic and nonpharmacologic therapies available for COPD. Tobacco cessation remains the most prominent intervention, as it is still the intervention that appears to have the greatest potential impact on disease progression. Little has changed regarding the recommendations concerning tobacco cessation approaches, with supportive counseling intervention supplemented by pharmacologic agents being the preferred mode of treatment. New in this edition of the report is an acknowledgment that electronic nicotine delivery devices (e-cigarettes) are a growing form of tobacco cessation therapy, but no recommendation is made as to their utility for this application, as evidence of their safety and efficacy for this application is, in GOLD's view, still indeterminate. ${ }^{13-15}$

The use of vaccinations receives a much stronger endorsement than in previous editions. The report presents data that the influenza vaccine can reduce the rate of exacerbations and other causes of hospitalization in the COPD population. ${ }^{16-18}$ This report is the first edition to examine the use of the 23-valent pneumococcal vaccine, and it endorses the recommendation of the Centers for Disease Control and Prevention Advisory Committee on Immuni- zation Practices to administer the 23-valent vaccine (in addition to the traditional 13 -valent vaccine) to all adults age 65 or older, as well as younger patients with risk factors or comorbid conditions that increase susceptibility to pneumonia, including COPD. ${ }^{19}$ GOLD bases their concurrence on data that show a reduction in the frequency of community-acquired pneumonia in relatively young ( $<65 \mathrm{y}$ of age) COPD patients having both grade 3 air flow obstruction category and comorbid diagnoses. ${ }^{20}$

The resources covering the pharmacologic armamentarium for COPD, including an overview table of medications and detailed descriptions of common drug classes, have been completely updated and are presented for reference. Research data for all classes of pharmaceuticals are again graded against the GOLD evidence-assessment criteria, and they reflect overall industry trends in questioning the role of chronic treatment with inhaled corticosteroids (ICS), especially as monotherapy. Roflumilast and/or macrolide antibiotic therapy is recommended for patients who endure frequent exacerbations, and the utility of less commonly used medications for COPD maintenance, such as mucolytics and antioxidants, is explored in detail. This provides a comprehensive, evidence-based reference guide to a variety of medications to assist in the creation and modification of management plans.

Nonpharmacologic therapies are of great interest in the latest GOLD report. These interventions take generally two forms: gas-related therapies (such as oxygen supplementation or noninvasive ventilation [NIV]) and mechanical alterations (surgical or bronchoscopic options). Oxygen therapy is perhaps the most common chronic, nonpharmacologic intervention for COPD patients, and it has been shown to improve outcomes for patients with 
severe resting hypoxemia (oxygen saturation by pulse oximetry $<88 \%$ on room air). ${ }^{21}$ The GOLD report incorporates the results of the recently concluded Long-Term Oxygen Treatment Trial ${ }^{22}$ and concurs that oxygen supplementation does not appear to improve long-term outcomes for patients with less severe hypoxemia at rest $\left(\mathrm{S}_{\mathrm{pO}_{2}} 89-93 \%\right)$ or induced by exercise $\left(\mathrm{S}_{\mathrm{pO}_{2}} 80-88 \%\right)$. Despite growing interest, the use of NIV as an out-patient maintenance therapy remains somewhat controversial, thus the new GOLD strategies remain equivocal on its use. The report declines to make any recommendations on NIV use, citing conflicting data from several randomized controlled trials regarding hospitalization rates and overall survival, but it does state that NIV may be beneficial for certain select patients when coupled with regular monitoring from a clinician trained in NIV use (such as a respiratory therapist).

A number of interventions aimed at reducing hyperinflation in regions of the heterogeneously emphysemic lung have been introduced over the last decade, and the GOLD report now includes details on these procedures to assist clinicians and patients in determining an appropriate course of action. Recommendations on these mechanical interventions have not changed to any significant degree, reflecting the difficulty in creating patient-selection criteria for procedures such as lung transplantation and lung volume reduction surgery. Similarly, newer, less invasive procedures such as the implantation of endobronchial valves or coils to evacuate areas of emphysemic parenchyma and allow re-expansion of healthier tissue suffer from a lack of long-term survival and efficacy data, in the view of the GOLD Science Committee. However, much as with the report's section on pharmacology, this information does represent a high-quality reference guide that enables RTs and others to present potential alternative therapies.

\section{Revised In-Patient and Out-Patient Management Strategies}

The most expanded section of the GOLD report concerns strategies for optimal ongoing disease management during the stable phase of COPD. In recognition of the role that exacerbations have as primary drivers of both cost and lung function decline in the COPD population, the GOLD report supports the notion that primary care providers (including RTs) must take a larger role in the routine outpatient management of the disease, focusing on both the reduction of everyday symptoms and the risk of exacerbations. Foremost in the GOLD strategy for routine maintenance is the identification and reduction of exposure to various environmental contaminants. These include not only obvious irritants like tobacco smoke, but also other forms of smoke and air pollution (indoor and outdoor), fumes, particulate matter, and occupational exposures. RTs must be actively involved in interviewing and screening patients for exposures to these contaminants at every patient encounter, especially with patients who are already being seen either in an emergency department or in-patient ward for an exacerbation.

Most patients with a COPD diagnosis are likely to utilize one or more pharmacologic agents as part of their disease maintenance regimen, and the report draws upon the updated drug reference section to provide several advisories and recommendations for use. As mentioned earlier, the strategy recommendations somewhat limit the role of ICS and remove them as a front-line therapy. This is stated to be due to the apparent increased risk of patients developing pneumonia while on ICS therapy (especially ICS monotherapy), ${ }^{23,24}$ evidence that the types of inflammation seen in COPD have a limited response to corticosteroid therapy in general according to in vitro models, ${ }^{25}$ and the potential for other adverse effects. ${ }^{26,27}$ The new recommendations have allowed the development of true disease management algorithms for COPD, similar to the stepwise approach commonly used in asthma treatment. GOLD has published an algorithm based directly on the Individualized Assessment; a similar, streamlined option using the same recommendations is presented in Figure 2.

The new strategy recommendations express an overt preference for long-acting muscarinic antagonists (LAMAs) over long-acting $\beta_{2}$-agonists (LABAs) in patients with relatively frequent exacerbations ( $\geq 2$ per year). GOLD cites two direct comparison studies (POET-COPD ${ }^{24}$ and INVIGORATE ${ }^{28}$ ) where the LAMA tested was superior to the LABA in terms of reduction in exacerbation frequency. LAMA/LABA combination therapy is preferred to LABA/ICS therapy in those with frequent exacerbations, again due to the enhanced risk of pneumonia inherent in ICS usage. The use of LAMA/LABA/ICS agents (triple therapy) is presented as an option, but the recommendations note that research into the relative efficacy of triple therapy compared to more basic regimens is still pending. Significant in this report is the recommendation to discontinue ICS use if no benefit is noted with regard to symptoms or exacerbation frequency. This is the first time that GOLD has gone beyond recommendations for initial treatment and the first time they have allowed for the deescalation of treatment in the absence of apparent efficacy. Roflumilast and macrolide antibiotics are options for those with frequent exacerbations along with significant symptom burdens. In patients with less frequent exacerbations, the strategy recommendations express no preference for either class of long-acting bronchodilator, although longacting agents are preferred to their short-acting counterparts.

Pulmonary rehabilitation programs take a prominent role in the new GOLD report. The new strategy notes that patients with COPD tend to be less physically active than 


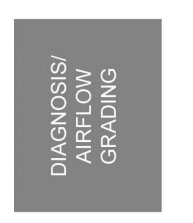

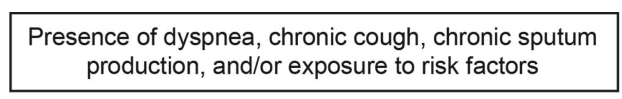

Spirometric confirmation $\left(\mathrm{FEV}_{1} / \mathrm{FVC}<0.70\right)$
- GOLD 1 ( $\mathrm{FEV}_{1} \leq 80 \%$ predicted)

- GOLD 2 (FEV $150-79 \%$ predicted)

- GOLD 3 (FEV $130-49 \%$ predicted)

GOLD $4\left(\mathrm{FEV}_{1}<30 \%\right.$ predicted $)$
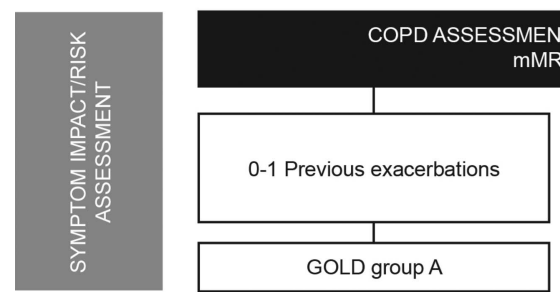

MENT TEST SCORE $<10$ MMRC 0-1
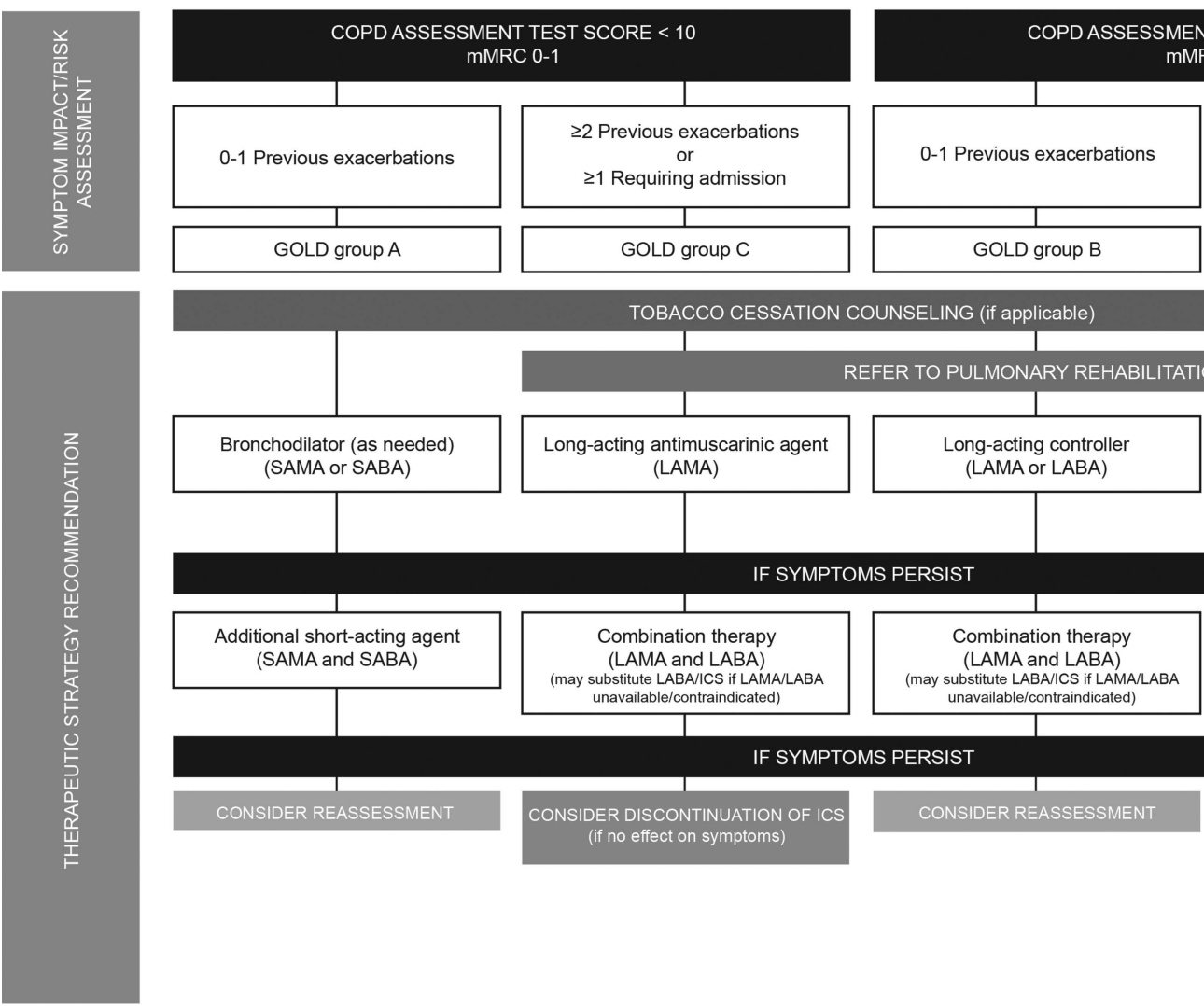

MENT TEST SCORE $\geq 10$ $\mathrm{mMRC} \geq 2$
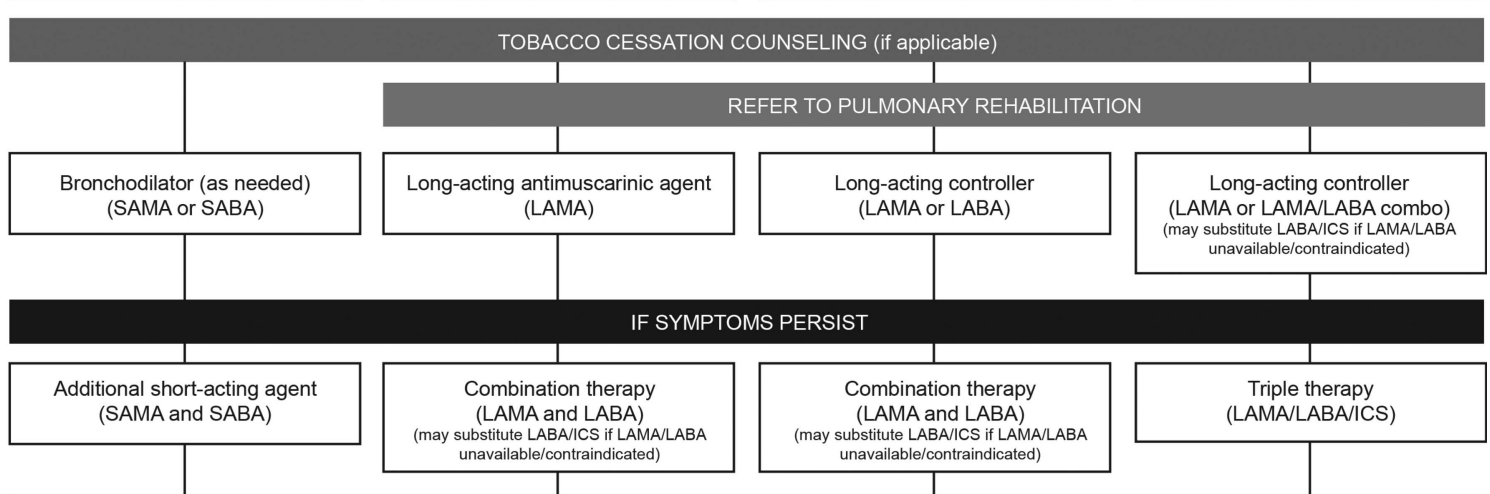

IF SYMPTOMS PERSIST
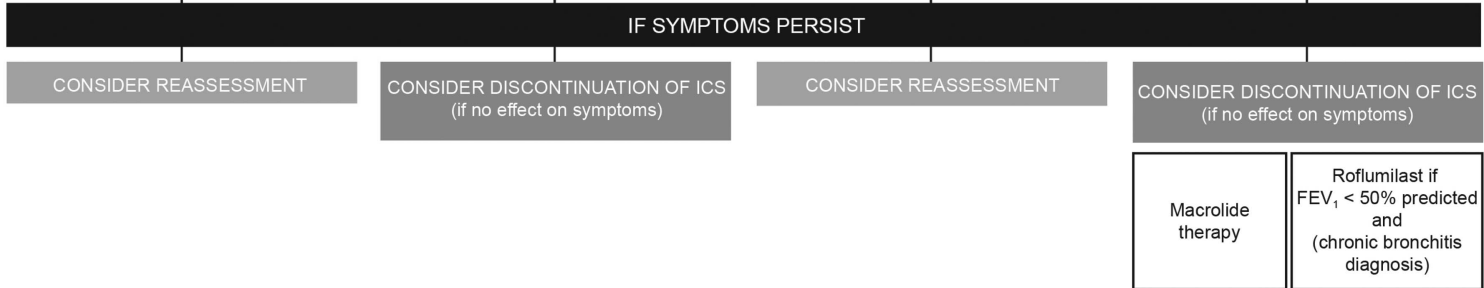

Fig. 2. Global Initiative for Chronic Obstructive Lung Disease (GOLD) 2017 diagnosis, assessment, and management algorithm. Note that previous exacerbations should be limited to the last 12 months. SAMA = short-acting muscarinic antagonist, SABA = short-acting $\beta$ agonist, LAMA = long-acting muscarinic antagonist, LABA = long-acting $\beta$ agonist, ICS $=$ inhaled corticosteroids, $\mathrm{mMRC}=$ modified Medical Research Council, CAT $=$ COPD Assessment Test.

their healthier counterparts, ${ }^{29}$ but a lack of research data hinders recommendations on specific frequency/intensity of exercise sessions or appropriate timing of exercise. In the absence of these factors, generalized pulmonary rehabilitation offers a reasonable solution. Pulmonary rehabilitation programs that are individualized to a patient's particular symptoms and comorbidities have been demonstrated to improve health status and subjective impact of symptoms. ${ }^{30}$ The GOLD report recommends all patients in Individualized Assessment groups B, C, and D be referred to rehabilitation and suggests that patients be referred either upon initial diagnosis, discharge from a COPD-related hospitalization, or when symptoms continue to deteriorate despite appropriate treatment. The report notes that the benefits of pulmonary rehabilitation wane over time and recommends that patients be encouraged to continue exercising after completion of a rehabilitation program (whether independently or as part of a maintenance regi- men). Recommendations for optimal exercise training routines are presented; however, these will vary based on resources available at each rehabilitation site and the specific capabilities of individual patients.

Patients with COPD will, despite optimal care, still experience exacerbations. There are virtually no new recommendations in this area since the previous update of the GOLD strategy document, reflecting a relative dearth of research progress in this area. The new report does note that existing data indicate that many mild exacerbations likely go unreported ${ }^{32}$ and emphasizes once again the critical nature of including symptom recognition in disease management education programs. In addition, to combat this failure to report and to facilitate better research concerning appropriate exacerbation treatment, the new report proposes stratifying patients into various exacerbation categories. The initial categorization of exacerbations is rated as mild, moderate, or severe, with mild exacerbations re- 
quiring only additional use of short-acting bronchodilators, moderate exacerbations adding antibiotic and/or oral corticosteroid agents, and severe exacerbations requiring an emergency department encounter and/or hospital admission. Severe exacerbations are further demarcated on the basis of whether symptom burden primarily reflects hypoxia, ventilatory insufficiency, or acute respiratory failure. Guidelines for proper classification are presented, and the relative benefits to pharmacologic and adjunct therapies are discussed. RTs involved in urgent care or emergency settings should become familiar with these guidelines to facilitate appropriate, evidence-based therapy as efficiently as possible, potentially avoiding a full admission. A longer period of post-acute transition follow-up care is suggested (12-16 weeks as compared to 4-6 weeks in previous reports), commensurate with the overall increased focus on out-patient management.

\section{New Horizons for Respiratory Therapists}

The new GOLD strategies launch at a time of enhanced interest in COPD in the United States health care community. The National Institutes of Health have recently released the first-ever COPD National Action Plan, which will become the foundation of COPD policy over the next decade. Much like the updated GOLD strategies, the National Action Plan calls for more intense out-patient and community-based disease management, recognizing that continuous management (rather than episodic treatment of exacerbations) will improve outcomes and enhance the efficiency of care delivery in this population. Respiratory therapists are well established providers of highly efficient, high-quality care. ${ }^{33-35}$ In addition, RTs are often acknowledged as subject matter experts in the area of pulmonary disease. Despite these endorsements, RTs are not commonly found in the out-patient space. Pathfinder programs utilizing RTs in case management, care coordination, and/or educational roles have been shown to provide improvements in readmission rates and/or resource utilization for organizations such as the Carolinas HealthCare System (http://www.hfma.org/leadership/COPD/, Accessed December 9, 2016) and the University of California-Davis Department of Internal Medicine (http:// www.ucdmc.ucdavis.edu/internalmedicine/pulmonary/ road-center.html, Accessed December 9, 2016), but these programs are not yet widespread. The renewed focus on cost reductions over reimbursement provides an opening for RTs to make a strong case for the aggressive expansion of these programs and deeper penetration into the primary care offices and community-based clinics.

The shift of COPD management to the out-patient world and development of ongoing education and self-management programs creates an opportunity for RTs to create personalized programs to maximize self-efficacy and op- timize self-care by the patient. The GOLD report addresses this need by suggesting key topics to cover for each Individualized Assessment classification. These may include behavioral risk factors (eg, smoking, indoor air quality issues, or the inadequate use of respirators/personal protective equipment), sleep hygiene, physical activity, and creation of an action plan. Covering these topics as part of a disease management education program engages the patient in their own care, enables them to better advocate for their own treatment, and empowers them to watch for signs of worsening disease. These plans should be tailored to the patient's individual history, comorbidities, personal preferences, and goals. When patients with COPD experience an exacerbation, it is an ideal time to identify not only environmental triggers, but failure points in a patient's management regimen. In-patient RTs are in an optimal position to recognize these issues and suggest therapy enhancements both during the hospital stay and after the transition to out-patient care.

The new standard of care for COPD includes knowledge areas not traditionally considered part of the RT scope, such as nutritional assessment. Systematic research reviews have indicated that appropriate nutrition is associated with improvements in exercise tolerance and ability, muscle strength, and subjective quality of life. ${ }^{31}$ It will be critical for RTs involved in pulmonary rehabilitation and patient education to add nutritional advice to their curricula, or at least to be able to recognize warning signs of malnutrition and refer patients to appropriate specialists for follow-up. The growing availability of palliative care programs, coupled with the current lack of lung function decline-arresting therapies, have led to the recommendation that palliative care consultation and appropriate end-of-life counseling be included throughout the COPD care continuum. RTs must be aware of resources in their local health care systems and in the general community that can provide patients with the information necessary to make informed, culturally appropriate decisions regarding the intensity of their ongoing care, and they should be prepared to facilitate active discussions regarding these decisions.

A recent survey of primary care providers (including physician assistants and nurse practitioners) demonstrated that roughly half of these providers do not use evidence-based guidelines (either GOLD or American Thoracic Society/ Eurpoean Respiratory Society) in their practice. ${ }^{36}$ A similar number reported the absence of spirometry in their practice. Interestingly, according to this survey, self-reported usage of COPD guidelines has increased since 2007, yet the presence of in-office spirometry has declined. ${ }^{36}$ This may represent an increase in referrals to specialty providers (at an increased cost to the patient), or over-reliance on complaints of COPDrelated symptoms when making a diagnosis; studies have indicated that perhaps as many as $66 \%$ of patients with air flow obstruction go without a spirometrically-confirmed 
COPD diagnosis, ${ }^{37}$ potentially because they have not complained of respiratory symptoms. In-office spirometry is a cost-effective diagnostic tool that can more accurately guide the initiation of appropriate therapy, and it can provide a standardized measure of disease progression. However, it is critical that whoever is performing the spirometry is a welltrained clinician (such as a respiratory therapist), as inadequate training can result in as many as $40 \%$ of tests being of insufficient quality for clinical use, and nearly 1 in 3 offering an outright misdiagnosis. ${ }^{38}$ RTs are ideally suited to recognize disconnects between objective clinical findings (such as spirometry values) and subjective symptom impact, and they must be prepared to recommend appropriate additional evaluation and/or diagnostic testing to the appropriate providers.

With the 2017 GOLD strategy report describing pulmonary rehabilitation referral appropriate for all but the least severe classification of patients with COPD, it is likely more physicians will seek pulmonary rehabilitation services for their patients. In recent years, access to pulmonary rehabilitation facilities has declined in response to trends in Medicare reimbursement rates. However, increased demand and adoption of evidence-based practice point toward the eventual expansion of pulmonary rehabilitation programs, which will increase the number of jobs available to qualified health care professionals, such as respiratory therapists.

One of the greatest opportunities presented to RTs is the GOLD report's new emphasis on self-management and education programs for the COPD patient, targeted at developing the skills necessary to manage disease independently. The new report marks the first time education and self-management have been focal points for COPD management, especially outside the context of pulmonary rehabilitation. The recommendations note that education alone is not sufficient to alter the natural progression of COPD, but patient education remains a key component of successful self-management programs, as enhancement of self-efficacy is a critical outcome of disease self-management. ${ }^{39}$ Improved self-efficacy in the context of COPD can have an impact on GOLD Individualized Assessment classification, as it has been linked to improvements in perceived breathlessness using the mMRC scale ${ }^{40}$ and reductions in exacerbations requiring hospitalization. ${ }^{41}$

Finally, the GOLD strategy's emphasis on nonpharmacologic and supportive treatment supports the introduction of RTs into new roles, and even into entirely new settings. For example, in geographic areas where pulmonary rehabilitation programs remain unavailable, disease management programs outside of traditional pulmonary rehabilitation may be more feasible or accessible. Such programs can be easily adapted for remote applications, especially when coupled with telemonitoring devices. Telemonitoring and teleconsultation have been demonstrated to be a cost-effective tool to reduce both clinic utilization and patient travel, ${ }^{42}$ important considerations for patients who are oxygen-dependent or have other difficulties with travel. Basic pulmonary rehabilitation techniques can be effectively delivered via remote connection, ${ }^{43}$ providing another avenue for RTs to contribute. Individual states are increasingly looking at telehealth as an efficient and effective adjunct to in-person care and are funding or reimbursing telehealth programs accordingly. As patients transition from the use of therapeutic interventions to more overtly palliative measures, RT services will be increasingly required in these settings. Palliative care, hospice services, and end-of-life discussions are prominent in the new GOLD report, and RTs can play a significant role in educating fellow clinicians, families and caregivers, and the patients themselves on what to expect as end of life approaches, as well as providing appropriate care throughout the process.

\section{Summary}

The latest iteration of the Global Strategy for the Diagnosis, Management, and Prevention of Chronic Obstructive Pulmonary Disease represents a shift in the way COPD is treated around the world. A heavier emphasis on enduring disease management, rather than episodic flare treatment, appears to present a significant opportunity for RTs to finally penetrate meaningfully into the out-patient care sector and provide diagnostic and patient education services alongside both primary care and specialty providers. The demand for pulmonary rehabilitation services is likely to increase, as pulmonary rehabilitation becomes even more integral to COPD treatment standards. RTs working in this specialty should expect increased patient loads, even if reimbursement levels remain in flux. However, as out-patient management increases in volume and improves in quality, it is likely that in-patient volume will decline (especially in non-intensive care settings). This lack of demand may negatively affect the work loads of traditional, in-hospital RT departments. It is unclear whether enhanced out-patient opportunity will fully balance any decrease in in-patient utilization. All RTs should familiarize themselves with the new GOLD diagnostic and disease management standards to capture patients who have gone undiagnosed or have been misdiagnosed, to maximize the demand for respiratory care services, to optimize outcomes for all respiratory patients, and to appropriately prepare themselves for inevitable shifts in health care delivery.

\section{REFERENCES}

1. World Health Organization. Chronic obstructive pulmonary disease (COPD) fact sheet. Geneva, Switzerland; WHO. 2016.

2. Thornton Snider J, Romley JA, Wong KS, Zhang J, Eber M, Goldman DP. The disability burden of COPD. COPD J Chronic Obstr Pulm Dis 2012;9(5):513-521. 


\section{GOLD 2017: IMPLICATIONS FOR RTS}

3. Guarascio AJ, Ray SM, Finch CK, Self TH. The clinical and economic burden of chronic obstructive pulmonary disease in the USA Clinicoecon Outcomes Res 2013;5:235-245.

4. Global Initiative for Chronic Obstructive Lung Disease (GOLD). Global strategy for the diagnosis, management and prevention of COPD. Global Initiative for Chronic Obstructive Lung Disease. 2016.

5. Vestbo J, Hurd SS, Agustí AG, Jones PW, Vogelmeier C, Anzueto A, et al. Global strategy for the diagnosis, management, and prevention of chronic obstructive pulmonary disease: GOLD executive summary (updated 2014). Global Initiative for Chronic Obstructive Lung Disease 2014;163(5):21-26.

6. Han MK, Muellerova H, Curran-Everett D, Dransfield MT, Washko GR, Regan EA, et al. GOLD 2011 disease severity classification in COPDGene: a prospective cohort study. Lancet Respir Med 2013; 1(1):43-50.

7. Jones PW. Health status and the spiral of decline. COPD 2009;6(1): 59-63.

8. Goossens LMA, Leimer I, Metzdorf N, Becker K, Rutten-van Mölken MPMH. Does the 2013 GOLD classification improve the ability to predict lung function decline, exacerbations and mortality: a posthoc analysis of the 4-year UPLIFT trial. BMC Pulm Med 2014; 14(1): 163 .

9. Kim JH, Yoon HI, Oh Y-M, Lim SY, Lee J-H, Kim T-H, et al. Lung function decline rates according to GOLD group in patients with chronic obstructive pulmonary disease. Int J Chron Obstruct Pulmon Dis Sept 2015;1819.

10. Martinez FJ, Mannino D, Leidy NK, Malley KG, Bacci ED, Barr $\mathrm{RG}$, et al. A new approach for identifying patients with undiagnosed chronic obstructive pulmonary disease. Am J Respir Crit Care Med 2017;195(6):748-756.

11. Wan ES, Castaldi PJ, Cho MH, Hokanson JE, Regan EA, Make BJ, et al. Epidemiology, genetics, and subtyping of preserved ratio impaired spirometry (PRISm) in COPDGene. Respir Res 2014;15(1): 89.

12. Vaz Fragoso CA, McAvay G, Van Ness PH, Casaburi R, Jensen RL, MacIntyre N, et al. Phenotype of normal spirometry in an aging population. Am J Respir Crit Care Med 2015;192(7):817-825.

13. Mcneill A, Brose LS, Calder R, Hitchman SC, Hajek P, H M. Ecigarettes: an evidence update: A report commissioned by Public Health England. Public Heal Engl 2015;111.

14. Hartmann-Boyce J, McRobbie H, Bullen C, Begh R, Stead LF, Hajek P. Electronic cigarettes for smoking cessation. In: Hartmann-Boyce J, ed. Cochrane Database of Systematic Reviews. Chichester, UK: John Wiley \& Sons, Ltd; 2016.

15. Schraufnagel DE, Blasi F, Drummond MB, Lam DCL, Latif E, Rosen MJ, et al. Electronic cigarettes: a position statement of the forum of international respiratory societies. Am J Respir Crit Care Med 2014;190(6):611-618

16. Wongsurakiat P, Lertakyamanee J, Maranetra KN, Jongriratanakul $\mathrm{S}$, Sangkaew S. Economic evaluation of influenza vaccination in Thai chronic obstructive pulmonary disease patients. J Med Assoc Thai 2003;86(6):497-508.

17. Nichol KL, Margolis KL, Wuorenma J, Von Sternberg T. The efficacy and cost effectiveness of vaccination against influenza among elderly persons living in the community. N Engl J Med 1994;331(12): 778-784.

18. Poole P, Chacko EE, Wood-Baker R, Cates CJ. Influenza vaccine for patients with chronic obstructive pulmonary disease. Cochrane Database of Systematic Reviews. Chichester, UK: John Wiley \& Sons, Ltd; 2006.

19. Kobayashi M, Bennett NM, Gierke R, Almendares O, Moore MR, Whitney CG, Pilishvili T. Intervals between PCV13 and PPSV23 vaccines: recommendations of the Advisory Committee on Immunization Practices (ACIP). MMWR Morb Mortal Wkly Rep 2015; 64(34):944-947.

20. Alfageme I. Clinical efficacy of anti-pneumococcal vaccination in patients with COPD. Thorax 2006;61(3):189-195.

21. Cranston JM, Crockett A, Moss J, Alpers JH. Domiciliary oxygen for chronic obstructive pulmonary disease. Cochrane Database of Systematic Reviews. Chichester, UK: John Wiley \& Sons, Ltd; 2005.

22. Albert RK, Au DH, Blackford AL, Casaburi R, Cooper JA Jr, Criner GJ, et al. A randomized trial of long-term oxygen for COPD with moderate desaturation. N Engl J Med 2016;375(17):1617-1627.

23. Crim C, Dransfield MT, Bourbeau J, Jones PW, Hanania NA, Mahler $\mathrm{DA}$, et al. Pneumonia risk with inhaled fluticasone furoate and vilanterol compared with vilanterol alone in patients with COPD. Ann Am Thorac Soc 2015;12(1):27-34.

24. Vogelmeier C, Hederer B, Glaab T, Schmidt H, Rutten-van Mölken MPMH, Beeh KM, et al. Tiotropium versus salmeterol for the prevention of exacerbations of COPD. N Engl J Med 2011;364(12): 1093-1103.

25. Barnes PJ. New anti-inflammatory targets for chronic obstructive pulmonary disease. Nat Rev Drug Discov. 2013;12(7):543-559.

26. Loke YK, Cavallazzi R, Singh S. Risk of fractures with inhaled corticosteroids in COPD: systematic review and meta-analysis of randomised controlled trials and observational studies. Thorax 2011; 66(8):699-708.

27. Johnell O, Pauwels R, Löfdahl CG, Laitinen LA, Postma DS, Pride $\mathrm{NB}$, Ohlsson S V. Bone mineral density in patients with chronic obstructive pulmonary disease treated with budesonide Turbuhaler. Eur Respir J 2002;19(6):1058-1063.

28. Decramer ML, Chapman KR, Dahl R, Frith P, Devouassoux G, Fritscher $\mathrm{C}$, et al. Once-daily indacaterol versus tiotropium for patients with severe chronic obstructive pulmonary disease (INVIGORATE): a randomised, blinded, parallel-group study. Lancet Respir Med 2013; 1(7):524-533.

29. Saunders T, Campbell N, Jason T, Dechman G, Hernandez P, Thompson K, Blanchard CM. Objectively measured steps/day in patients with chronic obstructive pulmonary disease: a systematic review and meta-analysis. J Phys Act Health 2016;13(11):1275-1283.

30. Corhay J-L, Dang DN, Van Cauwenberge H, Louis R. Pulmonary rehabilitation and COPD: providing patients a good environment for optimizing therapy. Int J Chron Obstruct Pulmon Dis 2014;9:27-39.

31. Ferreira IM, Brooks D, White J, Goldstein R. Nutritional supplementation for stable chronic obstructive pulmonary disease. Cochrane Database of Systematic Reviews. Chichester, UK: John Wiley \& Sons, Ltd; 2012.

32. Vijayasaratha K, Stockley RA. Reported and unreported exacerbations of COPD: analysis by diary cards. Chest 2008;133(1):34-41.

33. Shelledy DC, Legrand TS, Gardner DD, Peters JI. A randomized, controlled study to evaluate the role of an in-home asthma disease management program provided by respiratory therapists in improving outcomes and reducing the cost of care. J Asthma 2009;46(2): 194-201.

34. Kollef MH, Shapiro SD, Clinkscale D, Cracchiolo L, Clayton D, Wilner R, Hossin L. The effect of respiratory therapist-initiated treatment protocols on patient outcomes and resource utilization. Chest 2000;117(2):467-475.

35. Werre ND, Boucher EL, Beachey WD. Comparison of therapistdirected and physician-directed respir care in COPD subjects with acute pneumonia. Respir Care 2015;60(2):151-154

36. Yawn B, Wollan P, Textor K, Yawn R. Primary care physicians', nurse practitioners' and physician assistants' knowledge, attitudes and beliefs regarding COPD: 2007 to 2014. Chronic Obs Pulm Dis 2016;3(3):628-635. 


\section{GOLD 2017: IMPLICATIONS FOR RTS}

37. Johns DP, Walters JAE, Walters EH. Diagnosis and early detection of COPD using spirometry. J Thorac Dis 2014;6(11):1557-1569.

38. Hegewald MJ, Gallo HM, Wilson EL. Accuracy and quality of spirometry in primary care offices. Ann Am Thorac Soc. 2016;13(12):2119-2124.

39. Marks R. A review and synthesis of research evidence for selfefficacy-enhancing interventions for reducing chronic disability: implications for health education practice (part II). Health Promot Pract 2005;6(2):148-156.

40. Simpson E, Jones MC. An exploration of self-efficacy and selfmanagement in COPD patients. Br J Nurs 2013;22(19):1105-1109.
41. Laurin C, Moullec G, Bacon SL, Lavoie KL. Impact of anxiety and depression on chronic obstructive pulmonary disease exacerbation risk. Am J Respir Crit Care Med 2012;185(9):918-923.

42. Raza T, Joshi M, Schapira RM, Agha Z. Pulmonary telemedicine: a model to access the subspecialist services in underserved rural areas. Int J Med Inform 2009;78(1):53-59.

43. Stickland M, Jourdain T, Wong EYL, Rodgers WM, Jendzjowsky NG, Macdonald GF. Using telehealth technology to deliver pulmonary rehabilitation in chronic obstructive pulmonary disease patients. Can Respir J 2011;18(4):216-220. 